\title{
INTERNAL STRESS GENERATION DURING QUENCHING OF THICK HEAT TREATABLE ALUMINIUM ALLOYS
}

\author{
J-M. Drezet ${ }^{1}$, N. Chobaut ${ }^{1}$, P. Schloth ${ }^{1,2}$ and H. van Swygenhoven ${ }^{2}$ \\ ${ }^{1}$ Ecole Polytechnique Fédérale de Lausanne, Laboratoire de Simulation des Matériaux, \\ station 12, 1015 Lausanne, Switzerland \\ ${ }^{2}$ Paul Scherrer Institut, Materials Science and Simulation, ASQ/NUM, 5232 Villigen PSI, Switzerland
}

Keywords: Residual stress, neutron diffraction, precipitation, modeling

\begin{abstract}
In the current trend toward thicker aluminium plates, a major concern is the stress build-up during quenching which causes distortions during machining. Indeed, cooling rates are not high enough, especially at the core of such thick plates, to prevent any precipitation and quench induced precipitates lower the hardening potential. Multi-scale modelling is required when predicting macro-scale stresses after quenching for thick heat treatable aluminium components. The reason is the instantaneous strong coupling between phase precipitation at the nano-scale and material hardening due to precipitation or softening owing to solute depletion at the microscale. For thick parts, quenching intensities decrease when going from the skin to the core of the component, thus introducing a gradient of nanostructure and consequently a gradient of mechanical properties. In addition, large thermally induced deformations lead to high macroscale residual stresses although part of them is relaxed by plastic deformation. These stresses have been measured in water quenched thick plates of 7040 and 7449 aluminium alloys using neutron diffraction and layer removal techniques and the results when compared with a thermomechanical finite element model of quenching highlight the influence of precipitation.
\end{abstract}

\section{Introduction}

In the processing route of heat-treatable aluminium alloys (AA), a critical step is quenching from the solutionizing temperature. High cooling rates are desired, as they avoid formation of coarse precipitates, which reduce mechanical properties. Fast quenching, however, cannot be achieved in the centre of thick components, where the quenching rate can be more than one order of magnitude lower than at the surface. The existence of a thermal gradient between core and surface leads to a differential thermal deformation which is accommodated by elastic and plastic deformation to comply with the compatibility of deformation. Whenever the material yields, especially at high temperature when the yield strength is low, residual stress remains after quenching due to the irreversibility of plastic deformation. After quenching, the plates are in a state of residual stress that causes unacceptable distortions during machining and may reduce service life drastically. Since stress relaxation is negligible during ageing treatments [1], agehardened aluminium parts are usually stress relieved prior to ageing. This is typically achieved by applying plastic deformation: stretching from 1.5 to $3 \%$ in the rolling direction (L) can reduce the internal stresses by a factor of approximately 10 [2, 3]. However, complex geometries or thicker components like thick sheets require other approaches. Reducing the quenching rate by using boiling water for instance leads to lower residual stresses [1, 4]. For high quench sensitive 
alloys, however, slow quenching has the drawback to decrease the hardening potential. As a consequence, the ideal quench of heat treatable aluminium alloys remains a compromise between final properties and residual stresses.

Many researchers have developed thermo-mechanical models to predict stress build-up in thick aluminum alloy components [4-7]. To improve and validate the numerical models, a wide range of methods have been used for experimental measurement of residual stress: hole drilling, layer removal, crack compliance, ultrasounds, diffraction. While the agreement between experiments and simulations is good in the case of relatively thin components, thick components raise challenges. First, the numerical model must take metallurgical aspects into account for a reliable prediction of residual stresses since yield strength depends on precipitation state [6]. Second, the experimental method must be able to determine stresses deep inside the bulk. For thick components where long penetration paths are required, neutron diffraction has proved to be a suitable method as aluminium is rather transparent to neutrons [8]. Neutron diffraction has been applied successfully to the 7449 alloy by Robinson et al. [9] but the comparison with the deep hole drilling method indicated significantly different residual stress magnitudes due to the large residual stresses present in the forgings under study.

The purpose of the present contribution is to highlight the necessity of considering precipitation when predicting the stress generation during quenching of thick components. The first section presents the measurements of the residual stresses in as-quenched AA7040 and AA7449 plates. In the second section, the results of a thermo-mechanical model of quenching in which precipitation effects are ignored are compared with the measured stresses. In the last section, a microscopy characterization of the as-quenched nanostructure is presented and linked with the precipitation sequences reported for the 7xxx aluminum alloys.

\section{Residual stress measurements}

Before machining, finite element (FE) calculations were performed to determine the minimum plate dimensions in order to avoid side effects on residual stresses at the plate centre, as observed by Robinson et al. [9] on a 432mm (L, longitudinal, i.e rolling direction) $\times 156 \mathrm{~mm}$ (TL, long transverse) $\times 123 \mathrm{~mm}$ (TC, short transverse) block. The retained geometry of the as-quenched 75 $\mathrm{mm}$ thick plates provided by Constellium CRV is given in Figure 1. Assuming the plates are infinitely long in the $\mathrm{x}$ and $\mathrm{y}$ (in-plane) directions and no shear stresses are present, the problem reduces to a $1 \mathrm{D}$ plane stress problem (cf. equations 1 to 3 in figure 1 ) where only the in-plane stress component varies through the thickness, i.e. through the path $\mathrm{AB}$ in figure 1.

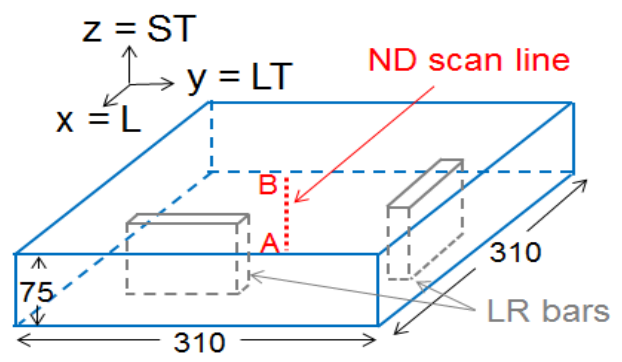

$$
\begin{aligned}
& \sigma_{x x}=\sigma_{y y} \\
& \sigma_{x y}=\sigma_{x z}=\sigma_{y z}=0 \text { no shear } \\
& \underline{\operatorname{div}}(\underline{\underline{\sigma}})=\underline{0} \Rightarrow \frac{\partial \sigma_{z z}}{\partial z}=0 ; \\
& \sigma_{z z}(-h)=\sigma_{z z}(h)=0 \Rightarrow \sigma_{z z}(z)=0
\end{aligned}
$$

Figure 1. Geometry of the plates for which stresses were measured at the centre through the thickness from A to B (left) and equations leading to the 1D plane stress problem. Bars for layer removal (LR) measurements of stresses are also shown (not at scale). 
FE calculations have shown that equations (1) to (3) are verified along the centerline (AB) of the plate. Although Chobaut et al. showed that texture inherent to hot rolling before solutionizing causes a decrease in intensity of the (311) reflexes, equation (1) was verified with neutron diffraction results [10]. An additional validation of equation (1) is presented in Fig. 2 reporting the residual stresses in a $75 \mathrm{~mm}$ thick AA7449 plate obtained with the destructive layer removal method performed by Constellium CRV. Two bars were cut from the plate in each direction (Fig. 1) and milled incrementally from top to bottom by steps of $2 \mathrm{~mm}$. A strain gauge attached to the rear face measures elastic deformations from which the residual stress components are calculated [11].

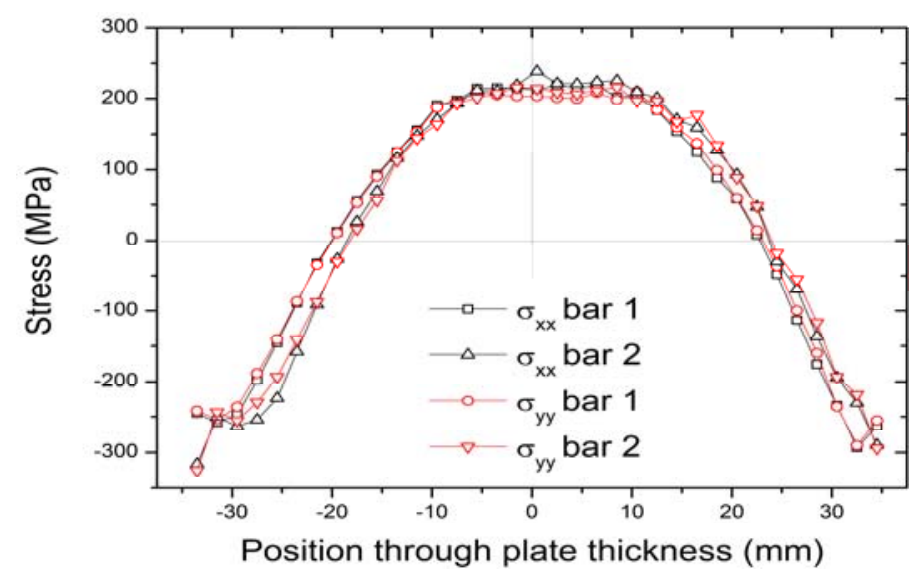

Figure 2. In plane stress distributions using the layer removal (LR) technique for the AA7449 plate (courtesy Constellium CRV).

Residual strains were measured by means of the time-of-flight neutron diffractometer POLDI [12] (Pulse-OverLap Diffractometer) located at the spallation source SINQ at the Paul Scherrer Institut in Villigen, Switzerland, and at the diffractometer SALSA [13] located at the Institut Laue-Langevin in Grenoble, France. While only one diffraction peak is recorded at SALSA usually corresponding to the (311) diffracting planes for fcc metals like aluminium - POLDI allows recording many peaks corresponding to several families of diffracting planes. This gives insight on texture and enables the calculation of an average lattice parameter using a full diffraction pattern Pawley analysis [14]. Strains are obtained by measuring with high accuracy the interplanar distance which acts as an internal strain gauge. Its variations causes changes in Bragg reflection peak positions. Stresses are calculated using the generalised Hooke's law, which writes:

$$
\sigma_{i j}=\frac{E}{1+v} \varepsilon_{i j}+\frac{E}{(1+v)(1-2 v)}(\operatorname{tr} \underline{\underline{\varepsilon}}) \delta_{i j}
$$

where $\underline{\underline{\varepsilon}}$ is the elastic strain tensor, $E$ the elastic modulus (71 MPa) and $v$ the Poisson's ratio (0.3). Using equations (1) to (3), eq. (4) simplifies to:

$$
\sigma_{x x}=\frac{E}{1-v} \varepsilon_{x x}
$$

This last equation, valid for an infinite plate without texture, means that the stress-state at the plate centre is fully determined by the measurement of only one elastic strain component.

The strain, $\varepsilon_{\mathrm{xx}}$, is given by: 


$$
\varepsilon_{x x}=\frac{d_{x}-d_{0}}{d_{0}}
$$

The reference value d0 is determined on small stress-free specimens machined out of the plate using EDM to take into account possible segregations. Chobaut et al. [10] have also shown that for such a simple geometry, d0 can be obtained non-destructively by measuring the interplanar distances in the $\mathrm{x}$ and $\mathrm{z}$ directions, $d_{x}$ and $d_{z}$, and by using all the equations above which give:

$$
d_{0}=\frac{2 v}{(1+v)} d_{x}+\frac{(1-v)}{(1+v)} d_{z}
$$

Hence, interplanar distances need to be measured in only $\mathrm{x}$ and $\mathrm{y}$ directions through the plate thickness from A to B (cf. Fig. 1) in order to determine the residual stress profile. The (311) diffraction peak was considered in all measurements presented in this paper. The results from SALSA using a gauge volume of $2 \times 2 \times 15 \mathrm{~mm}^{3}$ and from POLDI using a gauge volume of 3.8 x 6 x $1.5 \mathrm{~mm}^{3}$ are presented in figure 3 for the AA7040 and AA7449 plates, $75 \mathrm{~mm}$ in thickness. Results using the layer removal destructive technique at Constellium CRV are also added for the AA7449 plate. SALSA yields residual stresses about $10 \mathrm{MPa}$ higher than those given by POLDI which features a higher uncertainty. The stress profile measured using the layer removal technique is slightly shifted with respect to the thickness but the stress values at core and surface are close to the results obtained with neutron diffraction.

The overall agreement between experimental results using two different diffractometers and using the layer removal technique is excellent.

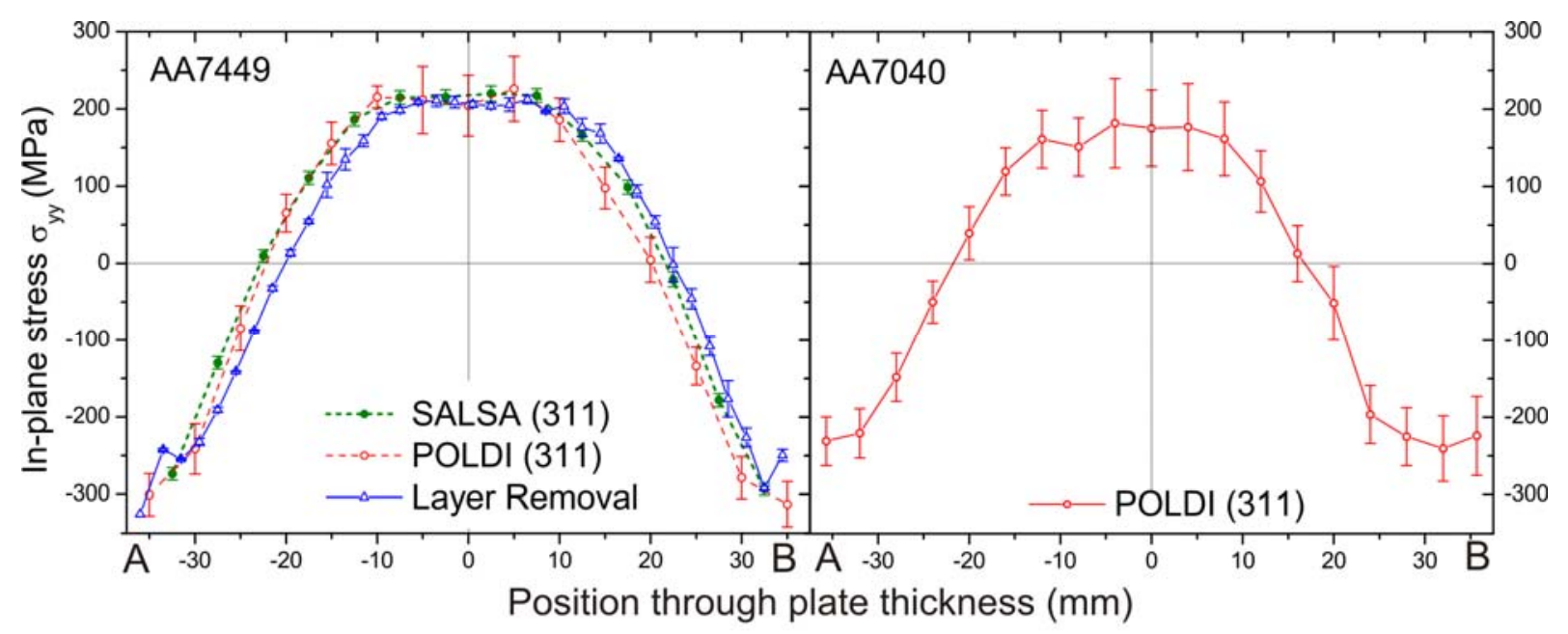

Figure 3. Through thickness residual stress profiles obtained at SALSA and POLDI for the AA7449 (left) and AA7040 (right) alloys.

The skin-core effect clearly appears with about $300 \mathrm{MPa}$ compressive stresses at the surface and $200 \mathrm{MPa}$ tensile stresses in the centre. Stresses are higher in absolute value at the surface than in the centre since the quenching rate is higher at the surface than in the centre [7, 15]. As expected, quench induced stresses are slightly lower in compression and in tension for the AA7040 alloy compared to the AA7449 alloy. These stress distributions will be used to validate thermomechanical models of quenching for both alloys.

Applying the global stress level indicator introduced by Heymes [11] to simplify the comparison between plates, the stored elastic energy per unit volume in a plate of thickness $2 \mathrm{~h}$ satisfying equations (1) to (3) writes: 


$$
W=\frac{1-v}{2 E h} \int_{-h}^{h} \sigma_{x x}^{2} d z
$$

Using the results of fig. 3, W is estimated as $225 \mathrm{~kJ} . \mathrm{m}^{-3}$ for the AA7040 and to $330 \mathrm{~kJ} . \mathrm{m}^{-3}$ for the AA7449 with a thickness of $75 \mathrm{~mm}$. AA7449 stores more energy than AA7040 since it contains more alloying elements available for strengthening. This elastic energy has to be lowered down, e.g. by stretching, to much lower values, around $2 \mathrm{~kJ} . \mathrm{m}^{-3}$, to avoid any significant distortion during machining according to aircraft manufacturers [11].

\section{Thermomechanical model of quenching}

The vertical quenching in cold water of an AA7449 aluminium thick plate was simulated using a thermo-mechanical model similar to the one used by Jeanmart and Bouvaist [4] and implemented in the commercial FE code ABAQUS 6.10. A temperature dependant heat transfer coefficient provided by Constellium was used and the mechanical behaviour of the AA7449 alloy was modelled with an elasto-viscoplastic law. The results of the simulation of cold water quenching of a $75 \mathrm{~mm}$ thick AA7449 plate are shown in Figure 4 together with the measured stresses.

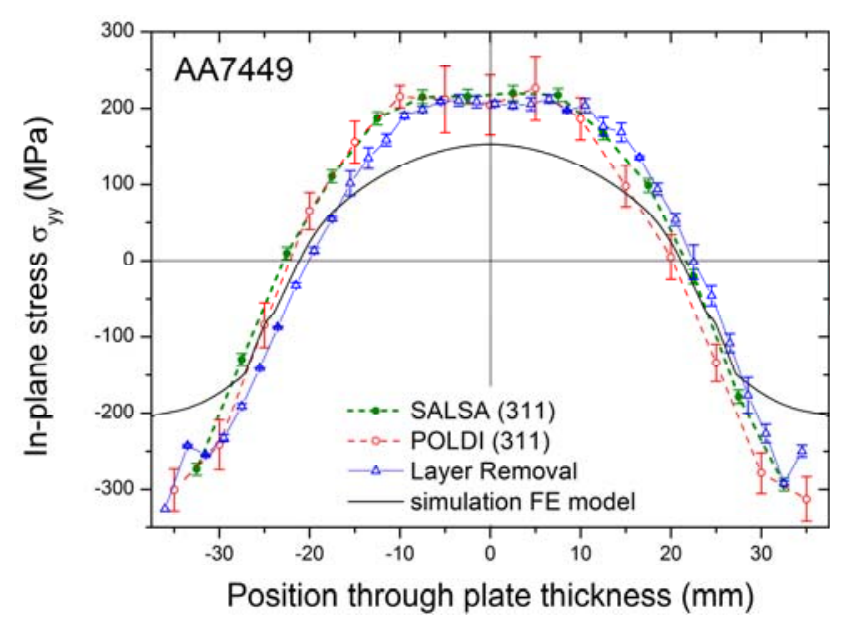

Figure 4. Residual in-plane stress distribution after quenching through as-quenched 75 mm thick AA7449 plates, comparison between numerical results and measurements.

The stress profile determined by FE simulation presents the same shape but different magnitude compared to the experimental one. Stresses determined numerically are systematically lower (35\% lower in the centre) in absolute value than those determined experimentally. This is explained by the fact that heterogeneous and homogeneous precipitation is ignored in the present model. Indeed quench induced precipitates increase or decrease the flow stress and hence affect plastic deformation and residual stresses. Such precipitation must be taken into account in the classic thermal-stress/strain coupling as schematically presented in figure 5. In the present case, this approach is multi-scale in the sense that the characteristics of the nanostructure largely influence the macro-stresses present in the plate after quenching. The temperature evolutions within the quenched part are used by a precipitation model to calculate the mean radius and volume fraction of precipitates. These values then feed a yield strength model aimed at defining the yield stress as a function of not only temperature but also thermal history. 


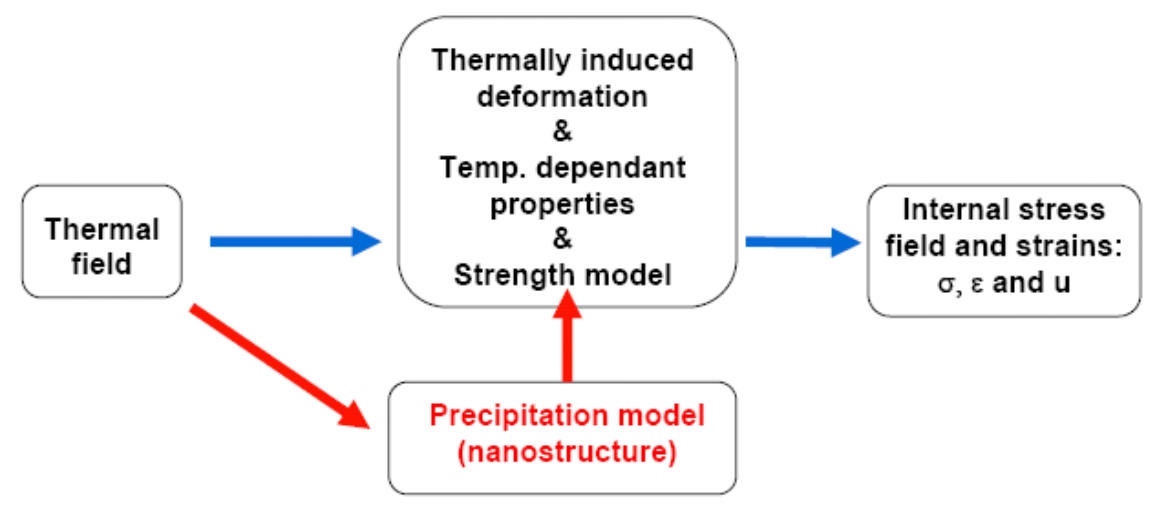

Figure 5. Schematics of the multi-scale coupling present in the stress generation during quenching of thick heat treatable alloy plates.

\section{Quench induced precipitation}

It is recognised that for quench sensitive alloys, quenching might induce some precipitation [16]. This is enhanced in the case of thick product where the cooling rate at the core of the part is not high enough to get a fully supersaturated solid solution.

During aging, the precipitation sequence of the 7xxx series alloys has been intensively studied and it is now accepted that the main precipitation sequence to be considered is SS (solid solution) $\rightarrow$ GP-zones $\rightarrow$ metastable $\eta$ ' $\rightarrow$ stable $\eta$ (MgZn2). The main hardening phase $\eta$ ' is present in the peak aged T6 state and has a variable composition. By using TEM, SEM and SAXS, Dumont et al. [17] have characterized the precipitation microstructures in the 7040 and 7050 aluminum alloys and highlighted the role of quench-induced precipitation on dispersoids. Deschamps et al. [18] have further investigated heterogeneous precipitation during quenching and showed that stable $\eta$ phase precipitates on dispersoids such as $\mathrm{Al}_{3} \mathrm{Zr}$ and on grain boundaries, using DSC measurements and TEM observations.

The influence of precipitation during quenching on stress generation has received little attention as only thin plates were studied so far. Nevertheless Godard [6] has studied the influence of precipitation in the AA7010 alloy during quench and has developed a thermomechanical model linking the solid-state transformations to the final stress distribution in the components using an additive elasto-visco plastic law. More recently, Fribourg [19] has studied the precipitation in the AA7449 alloy and the efficiency of thermomechanical treatments such as shot peening to reduce the distortions in aeronautical thin plates. Godard [6] has highlighted the complexity and variety of nucleation during quench of the AA7010 alloy. Nucleation can be homogeneous and heterogeneous (inter or intragranular). Grain and subgrain boundaries, dispersoids and even dislocations play a role. As during quenching high stresses develop, the alloy is plastically deformed and the associated dislocation density increase might influence nucleation during quench [20].

Figure 6 shows the as-quenched nanostructure of AA7449 samples using TEM, high angle annular dark field (HAADF) transmission electron microscopy and selected area diffraction pattern (SADP). Rather large $\eta$ precipitates, around $100 \mathrm{~nm}$ in size, are present at grain boundaries. They are also observed at dispersoids such as $\mathrm{Al}_{3} \mathrm{Zr}$, thus proving the heterogeneous nature of the precipitation of the stable phase. Much smaller $\eta$ ' precipitates, below 2 nanometers in size, are visible inside the grains together with Guinier-Preston zones (GPZ). 

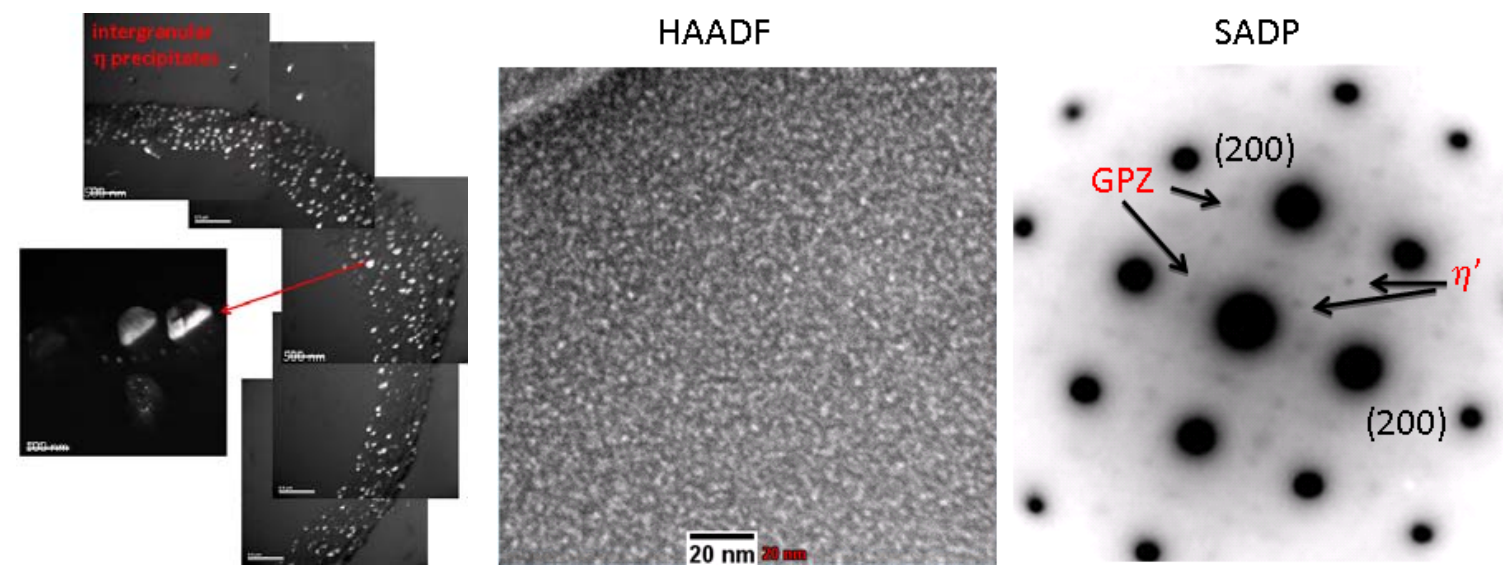

Figure 6. Nanostructure of AA7449 as-quenched samples: stable $\eta$ precipitates (left) and metastable $\eta$ ' precipitates (centre) together with GP zones (right).

Preliminary computations using the ThermoCalc database Al-data [21] and the Vircast precipitation model developed by Gandin and Jacot [22] have been carried out using three cooling curves, one at the surface of the part, one at the quarter thickness and one at the centre. The precipitation sequences are schematically summarized in figure 7. At high temperatures, stable $\eta$ phases nucleate and as they can become rather large they deplete the solid solution and are thus responsible for a softening of the material. On the other hand, at temperatures lower than $250^{\circ} \mathrm{C}$, both $\eta$ ' precipitates and GP zones appear and lead to a hardening effect owing to their very small size. These two opposite effects on the mechanical properties of the alloy during quenching must be taken into account in the modeling of the stress build up using a multi-scale model, as depicted in figure 5.

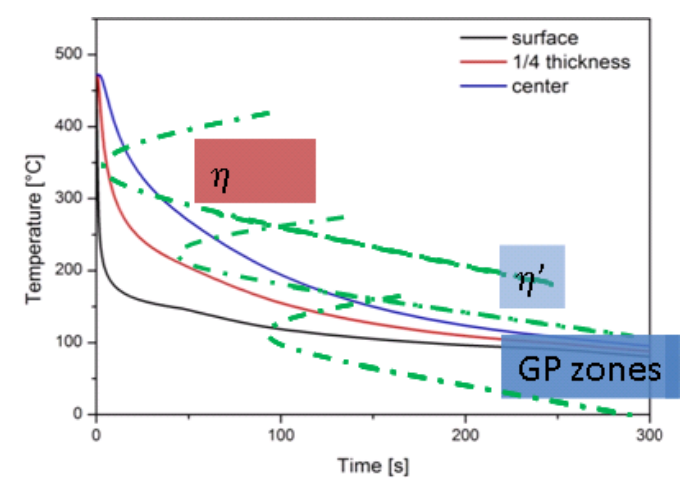

Figure 7. Schematics of the precipitation sequences during quenching of the AA7449 alloy in dependence of the cooling path.

\section{Conclusions}

The agreement between experimental determination of as-quenched stresses in thick AA7xxx alloy thick plates and FE simulation ignoring precipitation is poor. This is explained by the fact that quench induced precipitates have a major influence on the mechanical behavior of the alloy, notably on its yield strength. Microscopy observations of the as quenched nanostructure reveal the presence of both stable and metastable precipitates, which are in agreement with preliminary precipitation simulations. Such a multi-scale approach requires calibrating the input parameters 
entering both the precipitation and the yield strength models before reliable predictions of quench induced stresses can be obtained.

\section{Acknowledgement}

The authors are grateful to Constellium CRV, France, for the provision of samples and results using the layer removal technique, to the Swiss Spallation Neutron Source at PSI and the international neutron source at ILL for the provision of beam time. This work is funded by the Competence Center for Materials Science and Technology (http://www.ccmx.ch/) in the frame of the project entitled "Measurements and modelling of residual stress during quenching of thick heat treatable aluminium components in relation to their microstructure" involving EPF Lausanne, PSI Villigen, Univ. de Bretagne Sud Lorient, Constellium CRV and ABB Turbocharger.

\section{References}

1. Robinson, J.S. and D.A. Tanner, Materials Science and Technology, 2011.

2. Muammer, K., J. Culp, and A. Taylan, J. of Materials Processing Technology, 2006. 174.

3. Prime, M.B. and M.R. Hill, Scripta Materialia, 2002. 46(1): p. 77-82.

4. Jeanmart, P. and J. Bouvaist, Materials Science and Technology, 1985. 1(10): p. 765-769.

5. Drezet, J., The Minerals, Metals \& Materials Society. , 2010.

6. Godard, D.: Influence of precipitation on the thermomechanical behaviour in quenching an AlZn-Mg-Cu alloy, PhD thesis, Institut National Polytechnique de Lorraine, France, 1999.

7. Tanner, D.A. and J.S. Robinson, Journal of Materials Processing Technology, 2004.

8. J.-M. Drezet, A. Evans, T. Pirling and B. Pitié, Int. J. of Cast Metals, vol. 25, no. 2, March 2012, pp. 110-116.

9. Robinson, J.S., et al., Materials Science and Engineering: A, 2010. 527(10-11): p. 2603-2612. 10. N. Chobaut, J. Repper, T. Pirling, D. Carron and J-M. Drezet, International Conference on Aluminum Alloys (ICAA13), Edited by: Hasso Weiland, Anthony D. Rollett, William A. Cassada, TMS (The Minerals, Metals \& Materials Society),p. 285-291.

11. Heymès F. et al., $1^{\text {st }}$ Int. Non-Ferrous Processing and Technology Conf., 1997, St Louis, Missouri, USA.

12. Poldi, Pulse overlap time-of-flight diffractomer, Paul Scherrer Institut, Villigen, Switzerland, http://poldi.web.psi.ch/

13. Salsa, Strain imager for engineering applications, Institut Laue Langevin, Grenoble, France, http://www.ill.eu/instruments-support/instruments-groups/instruments/salsa/

14. Pawley, G.S., Journal of Applied Crystallography, 1981. 14: p. 357-361.

15. Li, P., et al., Metallurgical and Materials Transactions B, 2007. 38(4): p. 505-515.

16. Godard et al, Acta mater., vol. 50 (2002)

17. D. Dumont et al. in Materials Sc. and Tech., vol. 20, May 2004, p. 567-576.

18. A. Deschamps et al. in Materials Sc. and Eng. A 501 (2009) p. 133-139.

19. G. Fribourg, PhD thesis, Institut National Polytechnique de Grenoble, France, 2009.

20. A. Deschamps et. Acta Materialia 60 (2012) 1905-1916

21. AL-data base, Thermo-Calc Software, http://www.thermocalc.se/

22. Gandin et Jacot, Acta Mater., 55 (2007) 